\title{
Evidence of dispersion in an artificial water-saturated sand sediment
}

\author{
Preston S. Wilson ${ }^{\text {a) }}$ \\ Mechanical Engineering Department and Applied Research Laboratories, The University of Texas at Austin, \\ Austin, Texas 78712-0292 \\ Allen H. Reed \\ Seafloor Sciences Branch, Naval Research Laboratory, Stennis Space Center, Mississippi 39529-5004 \\ Jed C. Wilbur and Ronald A. Roy \\ Department of Aerospace and Mechanical Engineering, Boston University, Boston, Massachusetts 02215
}

(Received 2 February 2006; revised 3 November 2006; accepted 3 November 2006)

\begin{abstract}
A laboratory experiment was conducted to measure the speed of sound in an artificial water-saturated granular sediment composed of cleaned and sorted medium-grained sand and degassed distilled water. The experiment was conducted within a range of frequencies where dispersion is predicted by a number of existing models. Between 2 and $4 \mathrm{kHz}$, the sound speed was inferred from measurements of the resonance frequencies of a thin-walled cylindrical container filled with the material. An elastic waveguide model was used to account for the effect of the finite impedance of the walls, although this effect was found to be small. From 20 to $300 \mathrm{kHz}$, the sound speed was obtained directly from time-of-flight measurements within the sediment. Dispersion in close agreement with the Williams effective density fluid model [K. L. Williams, J. Acoust. Soc. Am. 110, 2276-2281 (2001)] was observed. (C) 2007 Acoustical Society of America.
\end{abstract}

[DOI: 10.1121/1.2404619]

PACS number(s): 43.30.Ma [RR]

Pages: $824-832$

\section{INTRODUCTION}

A complete understanding of sound propagation in water-saturated granular sediments has not been achieved. A number of experimental studies have appeared in the literature that report either direct or indirect evidence of sound speed dispersion in this medium. ${ }^{1-6}$ Such results generally support the Biot-Stoll model (and its variants) ${ }^{7-12}$ for sound propagation. The Biot-derived models predict a lowfrequency sound speed, followed by an increase to a higher sound speed beyond some transition frequency. There are also a number of experimental studies ${ }^{13,14}$ that found no evidence of sound speed dispersion and support Hamilton's dispersionless fluid model. ${ }^{15}$ The dispersion predicted by the Biot models occurs over a range of about two orders of magnitude in frequency (from around $100 \mathrm{~Hz}$ to $10 \mathrm{kHz}$ for medium-grained sand, for example). It is experimentally difficult to obtain measurements on a uniform body of sediment using a single technique that spans the entire range of interest. Further, the predicted dispersion is on the order of $10 \%$ and achieving experimental accuracy significantly greater than this has been difficult. Surface and volume inhomogeneities in natural sediments also cause uncertainty in experimental measurements. ${ }^{4,16}$

A series of laboratory experiments designed to overcome some of these difficulties were performed on a watersaturated sand sediment. The sediment was contained within a thin-walled cylindrical vessel large enough to permit time-

${ }^{a)}$ Electronic mail: pswilson@mail.utexas.edu of-flight sound speed measurements within the bulk sediment above $20 \mathrm{kHz}$. Lower frequency sound speeds were inferred from the measured resonance frequencies of the cylindrical sediment-filled container. For this analysis, transverse waves within the sediment were ignored. The sediment was modeled as a fluid, but the sediment sound speed was not constrained to be constant. The walls of the cylinder were initially approximated as a pressure release boundary (details of the assumption's validity are discussed in the following). The cylinder was placed upon a layer of insulation foam, which yielded a pressure release lower boundary condition. The water/air interface provided a pressure release boundary at the top of the sediment. Therefore, a pressure release waveguide model was used to relate the measured resonance frequencies to the effective sediment sound speed. In order to identify individual modes, and hence infer frequencydependent sound speeds from resonances, both symmetric and asymmetric excitation of the sediment cylinder was used. The spatially dependent phase of the acoustic pressure, relative to the drive signal, was employed to aid in mode identification. Dispersion was observed and these results are presented and compared to the effective density fluid model, ${ }^{12}$ hereafter referred to as EDFM. The effect of the finite impedance imparted by the cylindrical wall of the sediment container is also discussed. A model of an elasticwalled waveguide was used to relate the sediment sound speeds that were inferred from axisymmetric modes in the tank to the values that would be observed in an unconfined free field. The effect was found to be small (less than $0.53 \%$ ) at the lowest experimental frequency and decreased as the frequency increased. 


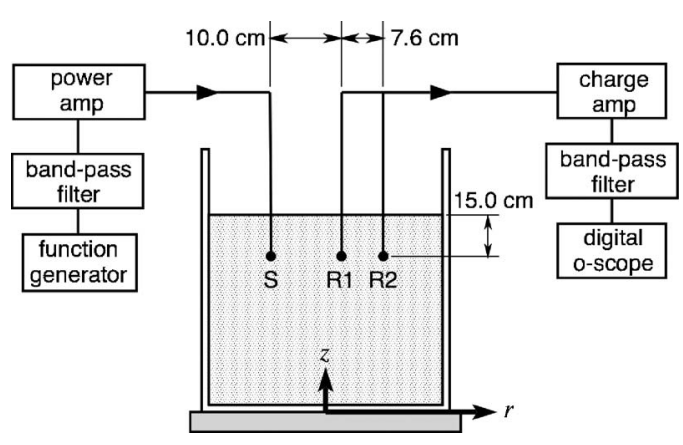

FIG. 1. A schematic diagram of the time-of-flight measurement apparatus.

\section{DESCRIPTION OF THE EXPERIMENT}

\section{A. Sediment container}

The sediment was contained in a right-circularcylindrical tank possessing walls made of high-density polyethylene (HDPE). The mean inner diameter was $0.686 \mathrm{~m}$, the height of the sediment within the vessel was $0.80 \mathrm{~m}$, and the mean wall thickness was $0.48 \mathrm{~cm}$. The tank, which had a flat bottom, was placed upon a 10 -cm-thick styrofoam panel which, in turn, rested on a concrete floor.

The sediment was prepared in the following manner. Filtered fresh water was added to the tank and degassed in situ by heating it to $60{ }^{\circ} \mathrm{C}$ using several immersion heaters. Circulation was imposed during this process with a motorized stirring impeller. The water was kept at $60{ }^{\circ} \mathrm{C}$ for several hours during which time the dissolved gas concentration attained equilibrium. Then, approximately $500 \mathrm{~kg}$ of a commercial silica blasting sand was slowly added to the water, as the stirring continued. The sediment was then allowed to cool to room temperature, over a period of $24 \mathrm{~h}$. As the system cooled, the water phase became undersaturated with gas and thus drew into solution any gas pockets entrained by the introduction of the sand, resulting in a fully saturated sediment. A sieve analysis conducted on approximately $500 \mathrm{ml}$ of the sand yielded grain sizes ranging from 150 to $600 \mu \mathrm{m}$, with a mean size of $300 \mu \mathrm{m}$.

\section{B. High-frequency time-of-flight measurements}

The sound speed within the sediment was measured using a time-of-flight technique at 20,50,100, and $300 \mathrm{kHz}$. A schematic diagram of this experiment is shown in Fig. 1. The signal source was a function generator programmed to produce sinusoidal pulses of various length, depending upon the frequency, to avoid multipath interference. The source signal was bandpass filtered ( $24 \mathrm{~dB} /$ octave) at the pulse center frequency with a Krohn-Hite model 34A filter and then amplified by a Krohn-Hite model 7500 wide-band power amplifier and directed to a Brüel \& Kjær model 8103 miniature hydrophone, operating as a source (labeled S in Fig. 1). Two more 8103 hydrophones were positioned 10.0 and $17.6 \mathrm{~cm}$ away from the source on a horizontal line (parallel to the sediment surface), at a depth of $15.0 \mathrm{~cm}$. The latter two hydrophones acted as receivers (labeled R1 and R2 in Fig. 1). The relative positions of the source and receivers were constrained to within $\pm 0.25 \mathrm{~mm}$ by mounting each hydrophone and its cable inside a stiff stainless steel tube. These tubes were

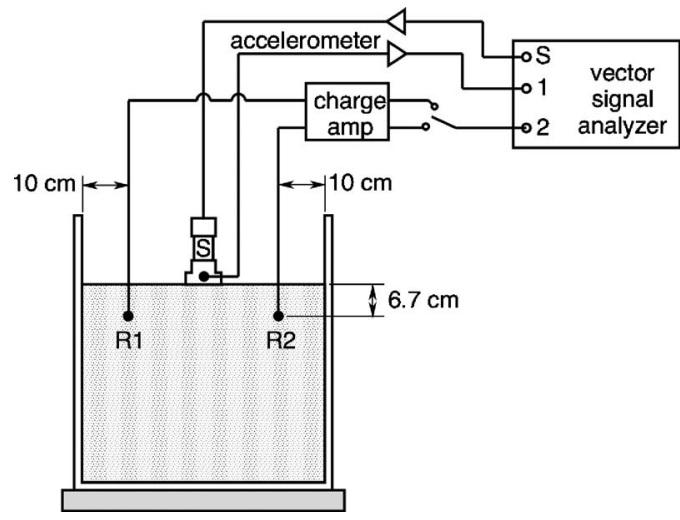

FIG. 2. A schematic diagram of the resonance frequency apparatus.

mounted to a steel frame and fastened securely in place. The R1-R2 separation distance was set and measured with a micrometer caliper prior to inserting the hydrophones into the sediment. The rigidity of the mounting scheme was sufficient to prevent caliper-measureable deflection during the inserting process. The hydrophone-enclosing tubes were filled with degassed water to minimize acoustic disturbance of the sediment.

The signals from R1 and R2 were conditioned with a Brüel \& Kjær model 2692 charge amplifier, then bandpass filtered (24 dB/octave) at the pulse center frequency with Krohn-Hite model 34A filters. The filtered source and receive signals were acquired with a digital oscilloscope. At each frequency, 100 pings were collected and the averaged signals were sent to a computer for processing. The R1 and R2 signals were cross-correlated to obtain the time-of-flight and then sound speed was calculated from the known sensor separation distance. The uncertainty in sound speed was calculated from the previously stated position uncertainty, and the digitization sample rate and found to be between \pm 6.6 and $\pm 9.9 \mathrm{~m} / \mathrm{s}$ at 300 and $20 \mathrm{kHz}$, respectively.

\section{Low-frequency resonance measurements}

The apparatus for this portion of the experiment is shown in Fig. 2. A custom-fabricated Tonpilz-type transducer was used as a source. It is described in Ref. 17 and has a circular-shaped radiating face with an accelerometer imbedded in it, and therefore can be driven at constant velocity amplitude by suitable monitoring of the accelerometer signal and modification of the excitation voltage amplitude. The source was placed face-down on the surface of the sediment in one of two positions, to be described later. The source signal was a band-limited $(2-5 \mathrm{kHz})$ periodic chirp, provided by a Hewlett-Packard 89410A vector signal analyzer (VSA) and amplified by a Crown CE 1000 power amplifier. The chirp amplitude was modified onboard the VSA to produce a source velocity amplitude that was uniform across the frequency range, as discussed earlier. The acoustic pressure was measured in the sediment with B\&K 8103 hydrophones at two positions, labeled R1 and R2 in Fig. 2. The depth was $6.7 \mathrm{~cm}$ and each sensor was positioned $10 \mathrm{~cm}$ from the vessel wall. When observed from above, R1-S-R2 formed a straight line. The signals from R1 and R2 were conditioned 
TABLE I. Specific acoustic impedance of sediment and tank materials.

\begin{tabular}{lc}
\hline \hline Material & Specific acoustic impedance $(\mathrm{Pa} \mathrm{s} / \mathrm{m})$ \\
\hline Sediment & $3.5 \times 10^{6}$ \\
Tank wall material, HDPE & $2.5 \times 10^{6}$ \\
Air & 415 \\
\hline
\end{tabular}

by the B\&K 2692 charge amp, which included bandpass filtering from 10 to $10 \mathrm{kHz}$, and digitized by the VSA. The complex spectrum of each hydrophone signal (with phase referenced to the excitation signal) was calculated onboard the analyzer using 100 spectral averages and converted to complex acoustic pressure spectra using the calibrated hydrophone sensitivities.

\section{ACOUSTIC MODEL OF SEDIMENT AND CONTAINER}

An acoustic model of the sediment/container system is needed to infer sound speeds from measured resonance frequencies. In this analysis, the sediment was considered to be an effective fluid with wave number $k$, which is a valid approach for granular sediments. ${ }^{12}$ We assume the phase speed can be frequency dependent, but $\operatorname{Im}[k] \ll \operatorname{Re}[k]$, so that the resonance frequencies depend primarily upon the phase speed and have weak dependence upon the attenuation. Transverse waves in the sediment were ignored.

Initially, we ignore the container walls and consider the sides of the cylinder to have a pressure-release boundary condition under the following justification. (1) Typical values of the specific acoustic impedance of the materials involved are given in Table I. Based on these values and the wall thickness $(0.48 \mathrm{~cm})$, the three-medium reflection coefficient $R$, given by Eq. 6.3.7 in Ref. 18 for pressure waves incident upon the wall, is $R=1.00 \angle 176.5^{\circ} \pm 1.5^{\circ}$ between 2 and $5 \mathrm{kHz}$. (2) This was qualitatively verified by the observation of inverted reflections from the walls during the time-offlight measurements, and (3) by the absence of plane-wave longitudinal mode resonances in the system. The airsediment interface at the top of the container is clearly pressure release. Finally, the container was placed upon a $10-\mathrm{cm}-$ thick styrofoam building insulation panel to approximate a pressure release boundary condition on the bottom. For the cylindrical coordinate system shown in Fig. 1 and sinusoidal excitation, the acoustic pressure in a right-circular cylinder with $p=0$ boundary conditions at all surfaces is

$$
\begin{aligned}
P_{m n N}(r, \theta, z, t)= & A_{m n N} J_{m}\left(\alpha_{m n} r / a\right) \\
& \times \cos \left(m \theta-\theta_{0 m}\right) \sin (N \pi z / h) e^{j \omega t},
\end{aligned}
$$

and has resonance frequencies

$$
f_{m n N}=\frac{c}{2} \sqrt{\left(\frac{\alpha_{m n}}{\pi a}\right)^{2}+\left(\frac{N}{h}\right)^{2}},
$$

where $\alpha_{m n}$ is the $n$th root of the Bessel function $J_{m}(x), c$ is the effective speed of sound in the sediment, $a$ is the tank radius, $h$ is the sediment height, $A_{m n N}$ is the modal amplitude coefficient, and $\theta_{0 m}$ is a phase angle which depends on the source condition. Equations (1) and (2) were adapted from

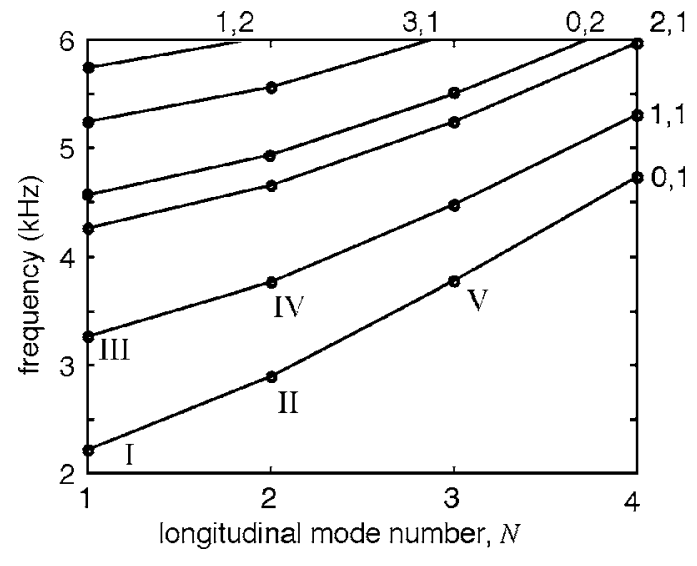

FIG. 3. The resonance frequencies for the six lowest-order mode families for a constant sound speed of $1730 \mathrm{~m} / \mathrm{s}$. The families are labeled $m, n$ around the top and right-hand side of the plot. The plane wave mode would be $0,0, N$ if it existed.

Ref. 19. A plot of resonance frequencies versus longitudinal mode number $N$ is shown in Fig. 3 for the six lowest-order mode families. Five modes, in order of ascending frequency, are labeled with roman numerals from I to V. Note that above mode III, multiple modes appear with similar eigenfrequencies.

Calculation of sound speed from a measured spectral peak requires one to identify the specific eigenmode and its $m, n, N$ values. Mode family $0,1, N$ is axisymmetric and mode family $1,1, N$ is asymmetric about the center of the sediment cylinder, as shown in Fig. 4. These two modes were selectively excited by using the source positions shown in Fig. 4. To excite mode family $0,1, N$, the source was placed at the center of the sediment cylinder. To excite mode family $1,1, N$, the source was placed halfway between the center and the container wall. The relative phase of the acoustic pressure observed at R1 and R2 was used to help identify the mode associated with an observed resonance peak. For example, Eq. (1) yields acoustic pressures at R1 and R2 that are in phase for modes I, II, and V, and $180^{\circ}$ out of phase for modes III and IV.

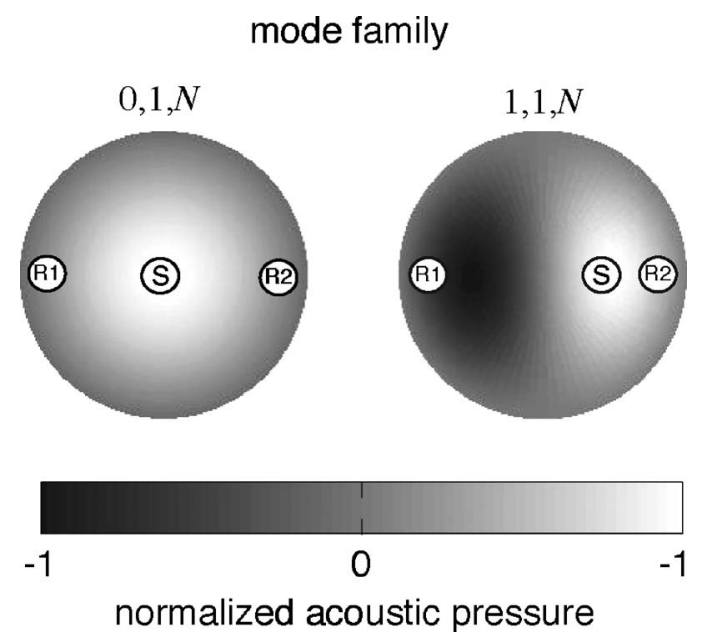

FIG. 4. The mode shapes given by Eq. (1) for two mode families. The source positions for each case are labeled $\mathrm{S}$. The receiver positions, which remain the same in both cases, are labeled $\mathrm{R} 1$ and $\mathrm{R} 2$. 


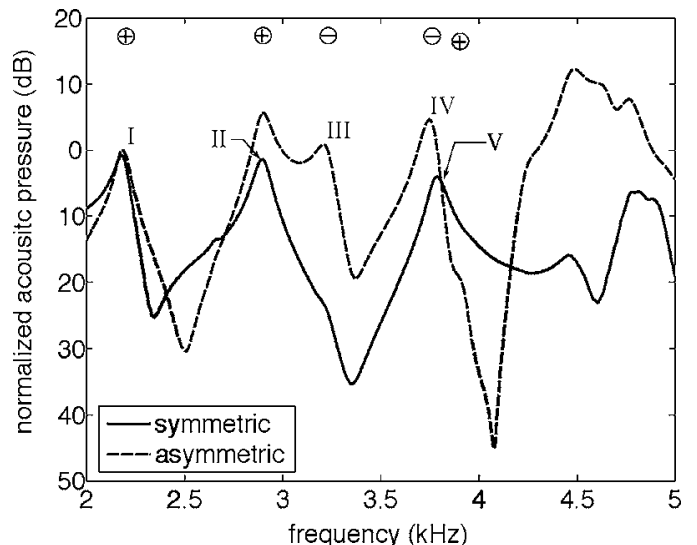

FIG. 5. Acoustic pressure spectra obtained at position R1 for both symmetric and asymmetric excitation. Spectra measured at position $\mathrm{R} 2$ were similar. The acoustic pressure measured between R1 and R2 was in phase (within $\pm 1^{\circ}$ ) at the resonance peaks labeled I, II, and V, and was out of phase (by $\left.170^{\circ}\right)$ at resonances III and IV, as indicated by $\oplus$ and $\ominus$.

\section{RESULTS}

A total of four averaged spectra were obtained during the resonance frequency experiment. These consisted of the acoustic pressure measured at R1 and R2, for both of the source positions shown in Fig. 4. The pressure spectra obtained at R1 are shown in Fig. 5. For the spectral peaks labeled I, II, and V, there was less than one degree phase difference between R1 and R2. These three spectral peaks are associated with mode family $0,1, N$ and correspond with the symmetric eigenmodes labeled I, II, and V in Fig. 3. Returning to Fig. 5, the spectral peaks labeled III and IV exhibited an R1-R2 phase difference of $170^{\circ}$. These peaks correspond to the asymmetric modes III and IV, shown in Fig. 3 and associated with mode family 1,1,N. Effective sediment sound speeds $c$ were calculated from each of the identified peaks in Fig. 5 using Eq. (2). Although not shown, similar results were obtained from the sensor at R2.

Both the resonance-based and time-of-flight-based sounds speeds are shown in Fig. 6. The speeds are normalized by the speed of sound in distilled water at the ambient temperature $\left(23.5 \pm 0.5^{\circ} \mathrm{C}\right)$. The sound speed predicted by the effective density fluid model ${ }^{12}$ is also shown in Fig. 6. The pertinent equations of the model are shown in the following for convenience. The sediment parameters used for the model calculation are shown in Table II. These were primarily taken from Ref. 12 but the following were obtained from measurements: The water sound speed was calculated from the experimental temperature. The porosity of four samples was determined by the water evaporation method. The permeability was measured using a Soiltest K-605 combination permeameter modified to accommodate a $13-\mathrm{cm}-$ long, 6.1-cm-diam core. Ten constant-head permeability tests ${ }^{20}$ were performed on each of two samples.

\section{SEDIMENT SOUND SPEED FROM THE EFFECTIVE DENSITY FLUID MODEL}

The effective density fluid model of sound propagation in water-saturated granular sediments is described in detail elsewhere, ${ }^{12}$ but the equations for prediction of the sediment

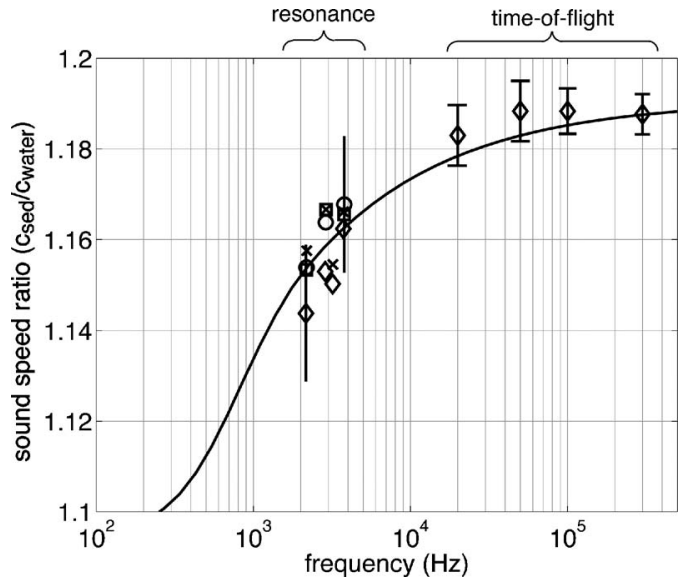

FIG. 6. Normalized sound speed measurements are compared to the EDFM (solid line). Resonance measurements are indicated with circles and squares (R1 and R2, respectively, with source in center) and crosses and diamonds (R1 and R2, respectively, source off-center, near R2). Vertical error bars near 2 and $4 \mathrm{kHz}$ represent sound speed uncertainty due to uncertainties in the dimensions of the tank, but are only shown for the minimum and maximum sound speed measurements, so that the remaining data points are not obscured. For the time-of-flight measurements, the error bars represent sound speed uncertainty due to uncertainty in the sensor separation distance and the time resolution of the data acquisition. The EDFM was evaluated using the parameters in Table II and the mean values of porosity and permeability.

sound speed are repeated here for convenience. The EDFM considers a water-saturated granular sediment with a total mass density $\rho$, pore fluid mass density $\rho_{f}$, sediment particle mass density $\rho_{s}$, individual sediment grain bulk modulus $K_{r}$, pore fluid bulk modulus $K_{f}$, porosity $\beta$, tortuosity $\alpha$, permeability $\kappa$, pore fluid viscosity $\eta$, and complex sound speed $c$. The sound speed is a function of angular frequency $\omega$ and is given by

$$
c=\sqrt{\frac{H}{\rho_{\mathrm{eff}}(\omega)}},
$$

where the compressibility is determined by a mixture rule

$$
H=\left(\frac{1-\beta}{K_{r}}+\frac{\beta}{K_{f}}\right)^{-1},
$$

and the effective density is

TABLE II. Material parameters used in the effective density fluid model (SI units). Parameters obtain from measurements on the sediment are in BOLDFACE. The mean value is followed by the range of variation among multiple measurements. The remaining parameters are from Ref. 12.

\begin{tabular}{lc}
\hline \hline Porosity & $0.370(0.368-0.371)$ \\
Sand grain mass density & 2650 \\
Water mass density & 998 \\
Sand grain bulk modulus & $3.6 \times 10^{10}$ \\
Water sound speed & 1492 \\
Water viscosity & 0.001 \\
Permeability & $4.58 \times 10^{-11}\left(4.18 \times 10^{-11}-4.74 \times 10^{-11}\right)$ \\
Tortuosity & 1.25 \\
\hline \hline
\end{tabular}


TABLE III. Material parameters used in the evaluation of Eq. (A1) (SI units).

\begin{tabular}{lr}
\hline \hline Sediment mass density, $\rho_{L}$ & 2039 \\
HDPE mass density, $\rho_{W}$ & 1000 \\
HDPE compressive sound speed, $c_{l}$ & 2500 \\
HDPE transverse sound speed, $c_{t}$ & 680 \\
\hline \hline
\end{tabular}

$$
\rho_{\mathrm{eff}}(\omega)=\rho_{f}\left(\frac{\alpha(1-\beta) \rho_{s}+\beta(\alpha-1) \rho_{f}+\frac{i \beta \rho F \eta}{\rho_{f} \omega \kappa}}{\beta(1-\beta) \rho_{s}+\left(\alpha-2 \beta+\beta^{2}\right) \rho_{f}+\frac{i \beta F \eta}{\omega \kappa}}\right) .
$$

The parameter $F$ represents a deviation from Poiseulle flow of the fluid within the pores and is given by

$$
F(\epsilon)=\frac{\frac{\epsilon}{4} T(\epsilon)}{1-\frac{2 i}{\epsilon} T(\epsilon)},
$$

where the Bessel functions $J_{\nu}(x)$ are used in

$$
T(\epsilon)=\frac{(-\sqrt{i}) J_{1}(\epsilon \sqrt{i})}{J_{0}(\epsilon \sqrt{i})}
$$

where the quantity $\epsilon=a \sqrt{\omega \rho_{f} / \eta}$ in conjunction with $a$ $=\sqrt{8 \alpha \kappa / \beta}$ represents the pore size parameter. The sound speed measurements shown in Fig. 6 are compared to the model phase speed given by real part of Eq. (3) and the material parameters of Table II.

\section{EFFECT OF FINITE WALL IMPEDANCE}

An exact analytic model ${ }^{21}$ for sound propagation in an elastic-walled, fluid-filled cylindrical tube was used to investigate the effects of finite wall impedance on the sound speeds inferred from the resonance frequency measurements for the axisymmetric modes. The model yields particle displacement field equations and a dispersion relation for the axisymmetric modes of an inviscid-liquid-filled cylindrical tube with arbitrary-thickness elastic walls, where the outermost radial boundary condition was approximated as pressure release. The dispersion relation has been verified for a variety of sizes and wall materials, including steel, ${ }^{17}$ aluminum, and $\mathrm{PVC}^{22}$ and is presented in Eq. (A1).

The unconfined (intrinsic) sound speed of the material that fills the tube is an input parameter to Eq. (A1) and the admissible phase speeds are calculated. This model is limited to axisymmetric modes, so we applied it to the $0,1, N$ modes and found that the maximum deviation of the sediment sound speed inferred from the pressure-release model was within $0.57 \%$ of the sound speed inferred from the elastic waveguide model and the minimum deviation was $0.18 \%$. The material parameters used for the evaluation of Eq. (A1) are given in Table III and the resulting sediment sound speeds are given in Table IV. The corresponding sediment sound speeds obtained with the pressure-release model are also included in Table IV for comparison.
TABLE IV. Comparison between sediment sound speed values $c$ of Fig. 6, which were inferred from the pressure-release model [Eq. (2)], and corrected sound speeds $c_{1}$, obtained from the axisymmetric elastic waveguide model [Eq. (A1)].

\begin{tabular}{lcc}
\hline \hline Frequency $(\mathrm{Hz})$ & $c(\mathrm{~m} / \mathrm{s})$, Eq. $(2)$ & $c_{1}(\mathrm{~m} / \mathrm{s})$, Eq. $(\mathrm{A} 1)$ \\
\hline \multicolumn{3}{c}{ Circle $(\circ)$ data points } \\
2179 & 1721 & 1712 \\
2892 & 1736 & 1731 \\
3788 & 1742 & 1739 \\
& Square $(\square)$ data points \\
2178 & 1721 & 1711 \\
2899 & 1741 & 1735 \\
3781 & 1739 & 1736 \\
2186 & Cross $(\times)$ data points \\
2899 & 1727 & 1717 \\
& 1741 & 1735 \\
2160 & Diamond $(\diamond)$ data points \\
2865 & 1706 & 1697 \\
\hline \hline
\end{tabular}

The relative size of the measurement uncertainty due to tank dimension uncertainty is compared to the finite wall impedance correction in Fig. 7. For clarity, this comparison is only shown for one of the data sets. The circle data points from Fig. 6 are repeated in Fig. 7 and their values after correction for the finite wall impedance are shown. The finite wall impedance biases the axisymmetric resonance measurements (circles and squares in Fig. 6) by $+0.57 \%$ or less, but this is significantly less than the measurement uncertainty due to the tank dimensions.

An exact analytic model for asymmetric excitation of a liquid-filled waveguide with walls of arbitrary thickness is not available. We used an approximate model ${ }^{23}$ which treats the wall as a shell and yields a dispersion relation Eq. (A6) for both axisymmetric and asymmetric modes. Otherwise, the corrections procedure is the same as for the axisymmetric case, discussed earlier. The dimensions of the sediment tank

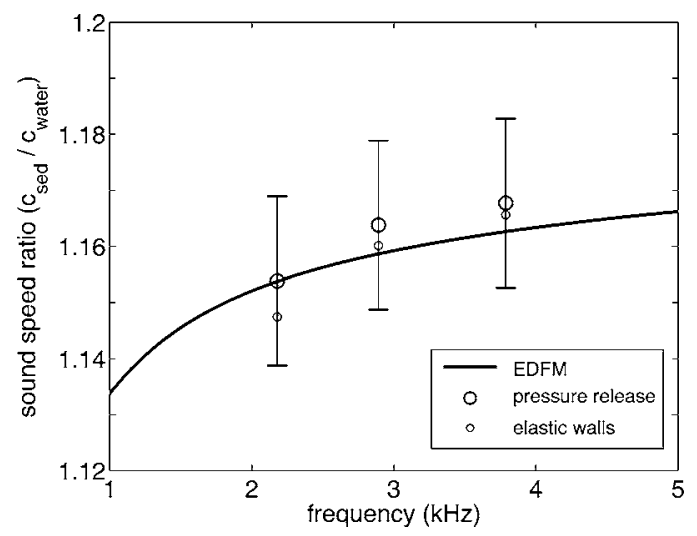

FIG. 7. The effect of ignoring the finite impedance of the tank walls is shown for the axisymmetric modes. The large circles are the sediment sound speeds inferred from a pressure-release model of the system, repeated here from Fig. 6, and the error bars represent the measurement uncertainty due to imperfect knowledge of the tank dimensions. The small circles are the sediment sound speeds inferred from a model which accounts for the finite impedance of the walls of the tank. Ignoring the finite impedance of the walls results in a maximum bias error of $+0.53 \%$ at the lowest frequency measured. 


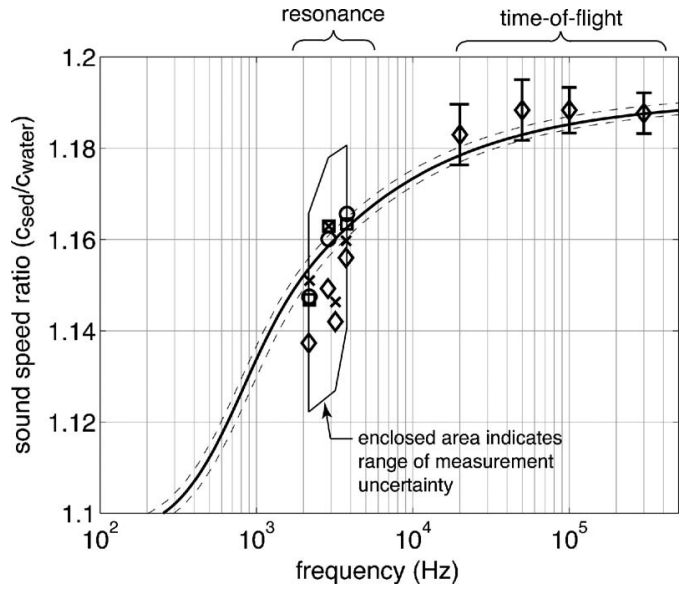

FIG. 8. The data and model prediction shown in Fig. 6 are repeated here, except that the resonance method measurements were corrected for the finite wall impedance of the tank. The extent of measurement uncertainty for the resonance-based measurements (due to sensor position and tank geometry uncertainty) is indicated by the closed polygon surrounding the data points. The solid curve is the EDFM prediction based on the mean porosity and mean permeability. The dashed curves above and below the solid curve represent the maximum and minimum EDFM predictions associated with the range of the porosity measurements and the standard deviation of the permeability measurements.

are well within the limits required by the shell approximation. To ensure continuity, we compared the phase speeds calculated by Eqs. (A1) and (A6) for the symmetric mode 0,1 and found excellent agreement. We are therefore confident that the approximate Eq. (A6) is accurate for the geometric and material properties used here. The data and model prediction presented in Fig. 6 are repeated in Fig. 8, but with the finite wall impedance correction applied to all the data from both axisymmetric and asymmetric modes. The asymmetric data before and after correction are presented in Table $\mathrm{V}$, and shows a maximum correction of $+0.76 \%$. Note that the time-of-flight measurements are not biased by the presence of the tank walls because the environment appears to be a free field for the pulse lengths and frequencies used.

\section{CONCLUSIONS}

Laboratory sound speed measurements were obtained in a water-saturated granular sediment composed of commercial silica blasting sand and fresh water. Above $20 \mathrm{kHz}$, a direct time-of-flight method was used and between 2 and $5 \mathrm{kHz}$, an indirect method was used to infer frequencydependent sound speeds from measured resonance frequen-

TABLE V. Comparison between sediment sound speed values $c$ of Fig. 6, which were inferred from the pressure-release model [Eq. (2)], and corrected sound speeds $c_{1}$, obtained from the axisymmetric elastic waveguide model [Eq. (A6)].

\begin{tabular}{lcc}
\hline \hline Frequency $(\mathrm{Hz})$ & $c(\mathrm{~m} / \mathrm{s})$, Eq. $(2)$ & $c_{1}(\mathrm{~m} / \mathrm{s})$, Eq. $(\mathrm{A} 6)$ \\
\hline \multicolumn{3}{c}{ Cross $(\times)$ data points } \\
3207 & 1723 & 1710 \\
3747 & 1740 & 1730 \\
3195 & Diamond $(\diamond)$ data points \\
3735 & 1716 & 1704 \\
\hline \hline
\end{tabular}

cies. The pressure-release approximation yields a surprisingly good model for a thin-walled plastic tank filled with a water saturated sediment, as shown in Figs. 6-8. There is at maximum about $0.5 \%$ error in sound speeds inferred from the pressure release model.

Considering the more realistic analysis which does account for the finite impedance of the tank walls and is shown in Fig. 8, the EDFM model ${ }^{12}$ describes the sound speed measurements very well. Most of the low-frequency measurements obtained with the resonance method are just lower than the predicted values, but the agreement between model and measurement is well within the measurement uncertainty. Three of the four high-frequency time-of-flight measurements are just higher than the prediction, but again, within the range of measurement uncertainty. The overall conclusion is that dispersion is clearly observed, as predicted by the Biot-derived models, and that the EDFM accurately describes the sound speed observed in this artificial laboratory sediment. Note that within the experimental frequency range, both measurements and model indicate about $4 \%$ dispersion. Evaluation of the EDFM from its low frequency asymptote $(\approx 100 \mathrm{~Hz})$ to its high frequency asymptote $(\approx 1 \mathrm{MHz})$ yields a total of about $8 \%$ dispersion. A secondary conclusion can be drawn from the spread in the resonance-inferred data, which sampled the entire sediment volume, as compared to the high frequency measurements which only sampled a small portion of the volume. Even in a carefully prepared laboratory specimen, granular sediments are acoustically nonhomogeneous.

Overprediction of sediment sound speed by Biot-type models in the transition region between the low and high frequency asymptotes has been previously reported in Refs. 1 and 4. One explanation for this deviation in the previous work is the existence of undissolved air. In the present experiment, significant effort was expended to achieve a gasfree sediment, and it appears that if there was any remaining undissolved air (in microscopic crevices for instance) the effect was not acoustically significant.

\section{ACKNOWLEDGMENTS}

This work was supported by the U.S. Navy Office of Naval Research Ocean Acoustics program, Boston University, The University of Texas at Austin, and the ONR Coastal Geosciences program. This is NRL Contribution No. NRL/ JA/7430-00-0014. The authors thank the reviewers for their astute suggestions.

\section{APPENDIX}

\section{Axisymmetric dispersion relation}

We considered sound propagation along the longitudinal axis of a lossless fluid cylinder enclosed by a lossless, arbitrary thickness, elastic cylindrical wall. Acoustic energy at angular frequency $\omega=2 \pi f$ propagating in this system (where $f$ is the number of cycles per second) is governed by a set of exact wave theoretic equations given originally by Del Grosso $^{21}$ and refined by Lafleur and Shields. ${ }^{22}$ The dispersion relation for this system is given in Eq. (A1). The ele- 
ments of Eq. (A1) can be complex valued, but real values of $q_{0 m}=\omega / c_{0 m}$ that cause the left-hand side of Eq. (A1) to become zero lead to propagating modes that have phase speed $c_{0 m}$. At any given frequency there can be multiple permissible modes, which are indexed by integer values of $m$. Since the phase speed is frequency-dependent, at a given sediment cylinder resonance frequency $f_{0, m, N}$, the phase speed will attain a value denoted by $c_{0, m, N}$. The zero subscript that precedes the index $m$ indicates that these are axisymmetric modes. The dispersion relation is

$$
\begin{aligned}
1+ & {\left[L_{11}\left(P_{m}\right) L_{00}\left(T_{m}\right)\right]\left(\frac{\pi^{2} q_{0 m}^{2} b d P_{m}^{2} T_{m}^{2}}{8 E_{m}^{2}}\right)+\left[L_{11}\left(T_{m}\right) L_{00}\left(P_{m}\right)\right]\left(\frac{\pi^{2} b d E_{m}^{2}}{8 q_{0 m}^{2}}\right)+\left[L_{10}\left(P_{m}\right) L_{01}\left(T_{m}\right)+L_{01}\left(P_{m}\right) L_{10}\left(T_{m}\right)\right] } \\
& \times\left(\frac{\pi^{2} b d P_{m}^{2} T_{m}^{2}}{8}\right)+\left[b L_{11}\left(P_{m}\right) L_{10}\left(T_{m}\right)+d\left(1+Q_{m} b\right) L_{11}\left(P_{m}\right) L_{01}\left(T_{m}\right)\right]\left(\frac{\pi^{2} P_{m}^{2} T_{m}}{8 E_{m}}-\frac{\pi^{2} P_{m}^{2} q_{0 m}^{2} T_{m}}{8 E_{m}^{2}}\right)+\left[b L_{11}\left(T_{m}\right) L_{10}\left(P_{m}\right)\right. \\
& \left.+d\left(1+Q_{m} b\right) L_{11}\left(T_{m}\right) L_{01}\left(P_{m}\right)\right]\left(\frac{\pi^{2} P_{m} E_{m}}{8 q_{0 m}^{2}}-\frac{\pi^{2} P_{m}}{8}\right)+\left[\left(1+Q_{m} b\right) L_{11}\left(T_{m}\right) L_{11}\left(P_{m}\right)\right]\left(\frac{\pi^{2} P_{m}}{8 q_{0 m}^{2}}-\frac{\pi^{2} P_{m}^{2} q_{0 m}^{2}}{8 E_{m}^{2}}-\frac{\pi^{2} P_{m}^{2}}{4 E_{m}}\right)=0,
\end{aligned}
$$

where the following definitions have been used:

$$
\begin{aligned}
& X_{0 m}=b \sqrt{k_{1}^{2}-q_{0 m}^{2}}, \quad P_{m}=\sqrt{k_{l}^{2}-q_{0 m}^{2}}, \quad T_{m}=\sqrt{k_{t}^{2}-q_{0 m}^{2}}, \\
& q_{0 m}=\omega / c_{0 m}, \quad k_{1}=\omega / c_{1}, \quad k_{l}=\omega / c_{l}, \quad k_{t}=\omega / c_{t}, \\
& E_{m}=q_{0 m}^{2}-k_{t}^{2} / 2, \quad Q_{m}=\frac{\rho_{L} \omega^{2} b J_{0}\left(X_{0 m}\right)}{2 \rho_{W} c_{t}^{2} X_{0 m} J_{1}\left(X_{0 m}\right)} \\
& L_{m n}(\xi)=J_{m}(d \xi) Y_{n}(b \xi)-J_{n}(b \xi) Y_{m}(d \xi),
\end{aligned}
$$

and the geometric and material input parameters are: $c_{1}$ is the intrinsic sound speed in the inner liquid (the sound speed that the sediment would exhibit in the free field), $c_{l}$ and $c_{t}$ are the longitudinal and transverse (shear) speed for the wall material, $\rho_{L}$ and $\rho_{W}$ are the liquid and wall material densities, and $b$ and $d$ are the inner and outer radii of the pipe, respectively. $J_{\nu}(x)$ and $Y_{\nu}(x)$ are Bessell functions of the first and second kind, respectively.

\section{Asymmetric dispersion relation}

We modeled nonaxisymmetric sound propagation along the longitudinal axis of a lossless fluid cylinder enclosed by a lossless, finite thickness, shell-like cylindrical wall, using the formulation of Fuller and Fahy. ${ }^{23}$ The dispersion relation is given in terms of a determinant

$$
\operatorname{det}\left[L_{i j}\right]=0
$$

where

$$
\begin{aligned}
& L_{11}=-\Omega^{2}+\left(k_{n m} b\right)^{2}+\frac{1}{2}(1-\nu) n^{2}, \\
& L_{12}=\frac{1}{2}(1+\nu) n\left(k_{n m} b\right), \quad L_{13}=\nu\left(k_{n m} b\right), \\
& L_{21}=L_{12}, \quad L_{22}=-\Omega^{2}+\frac{1}{2}(1-\nu)\left(k_{n m} b\right)^{2}+n^{2}, \quad L_{23}=n,
\end{aligned}
$$

$$
\begin{aligned}
& L_{31}=L_{13}, \quad L_{32}=L_{23}, \\
& L_{33}=-\Omega^{2}+1+\beta^{2}\left[\left(k_{n m} b\right)^{2}+n^{2}\right]^{2}-\mathrm{FL} .
\end{aligned}
$$

In Eq. (A7), $\Omega$ is the dimensionless frequency, $\Omega=\omega b / c_{l}, \nu$ is the ratio of Poisson of the wall material, $\beta$ is the wall thickness parameter $\beta^{2}=h^{2} / 12 b^{2}, h$ is the wall thickness, and FL represents fluid loading

$$
\mathrm{FL}=\Omega^{2}\left(\rho_{L} / \rho_{W}\right)(b / h)\left(1 / k_{m}^{r} b\right)\left[J_{n}\left(k_{m}^{r} b\right) / J_{n}^{\prime}\left(k_{m}^{r} b\right)\right] .
$$

The radial fluid wave number $k_{m}^{s} b$ and the axial wave number $k_{n m} b$ are related by

$$
k_{m}^{r} b=\left[\Omega^{2}\left(c_{l} / c_{1}\right)^{2}-\left(k_{n m} b\right)^{2}\right]^{1 / 2} .
$$

\section{Illustration of finite wall impedance correction scheme}

The effect of the finite wall impedance and the method of correction for this effect are demonstrated in Fig. 9 for the axisymmetric mode. The phase speed for the $0,1, N$ mode is shown for both the pressure release model and the elastic waveguide model Eq. (A1). Note that the predicted phase speeds are similar, therefore we do not expect the corrections to be large. For a given experimentally observed resonance frequency $f_{0,1, N}$, the observed modal phase speed is calculated from

$$
c_{0,1, N}=\lambda f_{0,1, N},
$$

where $\lambda=2 h / N$. Then the effective sediment sound speed $c_{1}$ is varied in Eq. (A1) until the predicted modal phase speed is $c_{0,1, N}$. This procedure was repeated for each resonance frequency identified from an axisymmetric mode. The correction procedure is identical for the asymmetric modes, except Eq. (A1) is replaced by Eq. (A6). The results are reported in Table IV and shown in Fig. 8. 


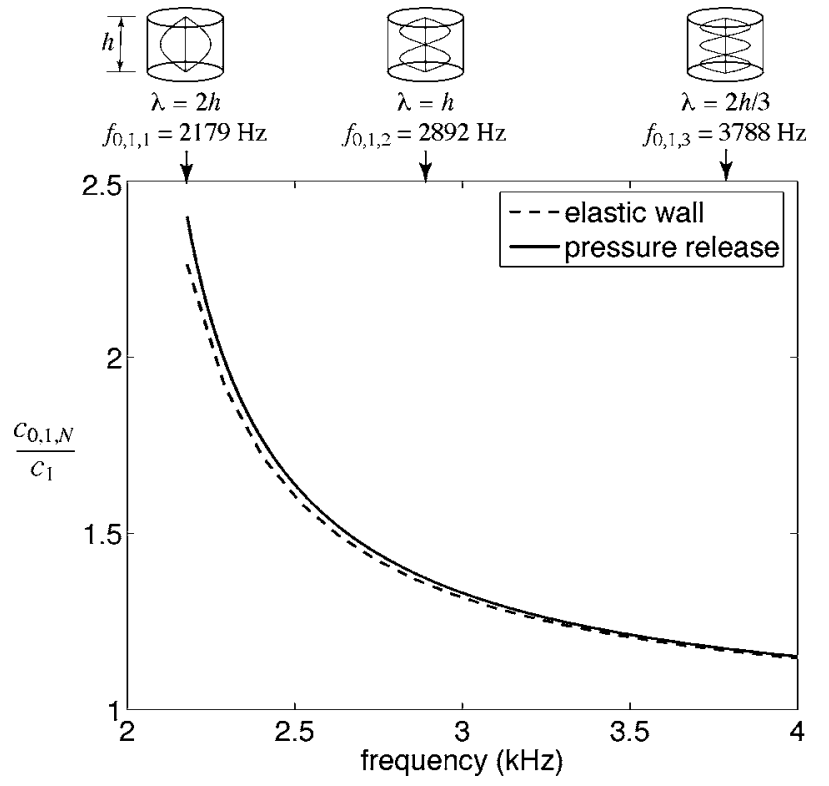

FIG. 9. The effect of the finite wall impedance on the modal phase speed $c_{0,1, N}$. The vertical axis is normalized by the sediment effective intrinsic sound speed $c_{1}$. The basis for the procedure used to correct for the finite wall impedance is illustrated for the one of the data sets (the open circles of Fig. 6). Integer multiples of one-half wavelength occupy the sediment cylinder along its height for the $0,1, N$ modes. Knowledge of these mode shapes from Eq. (1), the measured resonance frequencies $f_{0,1, N}$ and Eq. (A10) allow one to use Eq. (A1) to relate the measured value of $c_{0,1, N}$ to the intrinsic effective sediment sound speed $c_{1}$ that would be measured in the free field. The results of the correction are shown in Table IV.

\section{Effect of neglecting viscosity in the wall impedance correction}

The above-presented elastic waveguide models approximate the internal fluid as inviscid, therefore the fluid can slip at the boundary with the wall. In this section it is shown that neglecting the sediment viscosity in the waveguide models has an insignificant effect on the phase speeds in this experiment. This is due to the large size of the sediment tank in comparison to the boundary layer at the wall.

In this work, the sediment is approximated as an effective fluid and can be assigned an effective viscosity with respect to pipe wall interaction, as is done in the modeling of slurry flows, ${ }^{24}$ and in acoustic studies of sediments in tubes. ${ }^{25}$ For mixtures of liquids and sand with high solid volume concentrations (such as the present sediment) the effective mixture viscosity $\mu_{\text {mix }}$ is dependent upon the solid volume concentration $\phi$ as

$$
\frac{\mu_{\mathrm{mix}}}{\mu}=1+2.5 \phi+10.05 \phi^{2}+2.73 \times 10^{-3} \exp (16.6 \phi),
$$

where $\mu$ is the viscosity of the host liquid. ${ }^{24}$ The sediment porosity in this work is 0.37 therefore $\phi=0.63$ and Eq. (A11) yields a viscosity ratio $\mu_{\text {mix }} / \mu=102$.

For acoustic waves propagating within a viscous fluid inside a pipe, an acoustic boundary layer is formed at the wall. The resulting acoustic effects can be modeled with a complex wave number ${ }^{26}$

$$
\tilde{k}=\frac{\omega}{c}+(1-i) \alpha_{\text {wall }},
$$

where $c$ is the effective sound speed of the sediment inside the waveguide and $\omega / c \gg \alpha_{\text {wall }}$ is assumed. Thermal losses are considered negligible compared to the viscous losses, which are characterized by

$$
\alpha_{\mathrm{wall}}=\frac{1}{a} \sqrt{\frac{\mu_{\mathrm{mix}} \omega}{2 \rho c^{2}}}
$$

where $a$ is the inner radius of the pipe and $\rho$ is the total mass density of the sediment. Equation (A12) indicates a reduced phase speed inside the waveguide due to the viscous boundary layer. The effect of the viscosity on phase speed can be expressed in a ratio

$$
\frac{\widetilde{c}}{c}=\left(1+\frac{c \alpha_{\text {wall }}}{\omega}\right)^{-1}
$$

where $\tilde{c}=\operatorname{Re}[\omega / \tilde{k}]$ is normalized by the sound speed in absence of a viscous boundary layer, $c$.

Using the sediment mass density from Table III, the highest frequency in the resonance regime of the experiment $(3788 \mathrm{~Hz})$ and the mean sound speed observed inside the waveguide at the same frequency $(1740 \mathrm{~m} / \mathrm{s})$, Eq. (A14) yields $\widetilde{c} / c=0.99905$, which is a negligible systematic error in comparison to other sources of measurement uncertainty in this experiment and is ignored.

${ }^{1}$ A. Turgut and T. Yamamoto, "Measurement of acoustic wave velocities and attenuation in marine sediments," J. Acoust. Soc. Am. 87, 2376-2383 (1990).

${ }^{2}$ A. Maguer, E. Bovio, W. L. J. Fox et al., "In situ estimation of sediment sound speed and critical angle," J. Acoust. Soc. Am. 108, 987-996 (2000). ${ }^{3}$ I. Rosenfeld, W. M. Carey, P. G. Cable et al., "Modeling and analysis of sound transmission in the Strait of Korea," IEEE J. Ocean. Eng. 26, 809820 (2001).

${ }^{4}$ K. L. Williams, D. R. Jackson, E. I. Thorsos et al., "Comparison of sound speed and attenuation measured in a sandy sediment to predictions based on the Biot theory of porous media," IEEE J. Ocean. Eng. 27, 413-428 (2002).

${ }^{5}$ R. D. Stoll, "Velocity dispersion in water-saturated granular sediment," J. Acoust. Soc. Am. 111, 785-793 (2002).

${ }^{6}$ N. P. Chotiros, A. P. Lyons, J. Osler et al., "Normal incidence reflection loss from a sandy sediment," J. Acoust. Soc. Am. 112, 1831-1841 (2002). ${ }^{7}$ M. A. Biot, "Theory of propagation of elastic waves in a fluid-saturated porous solid. I. Low-frequency range," J. Acoust. Soc. Am. 28, 168-178 (1956).

${ }^{8}$ M. A. Biot, "Theory of propagation of elastic waves in a fluid-saturated porous solid. II. Higher frequency range," J. Acoust. Soc. Am. 28, 179191 (1956).

${ }^{9}$ R. D. Stoll and T. K. Kan, "Reflection of acoustic waves at a watersediment interface," J. Acoust. Soc. Am. 70, 149-156 (1981).

${ }^{10}$ N. P. Chotiros, "Biot model of sound propagation in water-saturated sand," J. Acoust. Soc. Am. 97, 199-214 (1995).

${ }^{11}$ M. J. Buckingham, "Wave propagation stress relaxation and grain-to-grain shearing in saturated unconsolidated marine sediments," J. Acoust. Soc. Am. 108, 2796-2815 (2000).

${ }^{12} \mathrm{~K}$. L. Williams, "An effective density fluid model for acoustic propagation in sediments derived from Biot theory," J. Acoust. Soc. Am. 110, 22762281 (2001).

${ }^{13}$ E. L. Hamilton, "Geoacoustic modeling of the sea floor," J. Acoust. Soc. Am. 68, 1313-1340 (1980).

${ }^{14}$ E. L. Hamilton and R. T. Bachman, "Sound velocity and related properties of marine sediments," J. Acoust. Soc. Am. 72, 1891-1904 (1982).

${ }^{15}$ E. L. Hamilton, "Prediction of in-situ acoustic and elastic properties of marine sediments," Geophysics 36, 266-284 (1971). 
${ }^{16}$ R. D. Stoll, G. M. Bryan, and E. O. Bautista, "Measuring lateral variability of sediment geoacoustic properties," J. Acoust. Soc. Am. 96, 427-438 (1994).

${ }^{17}$ P. S. Wilson, R. A. Roy, and W. M. Carey, "An improved water-filled impedance tube," J. Acoust. Soc. Am. 113, 3245-3252 (2003).

${ }^{18}$ L. E. Kinsler, A. R. Frey, A. B. Coppens et al., Fundamentals of Acoustics, 4th ed. (Wiley, New York, 2000).

${ }^{19}$ D. T. Blackstock, Fundamentals of Physical Acoustics (Wiley, New York, 2000).

${ }^{20}$ ASTM, Standard Designation D 2434-68: Standard Test Method for Permeability of Granular Soils (American Society for Testing and Materials, West Conshohocken, PA, 2006).

${ }^{21}$ V. A. Del Grosso, "Analysis of multimode acoustic propagation in liquid cylinders with realistic boundary conditions-Application to sound speed and absorption measurements," Acustica 24, 299-311 (1971).

${ }^{22}$ L. D. Lafleur and F. D. Shields, "Low-frequency propagation modes in a liquid-filled elastic tube waveguide," J. Acoust. Soc. Am. 97, 1435-1445 (1995).

${ }^{23}$ C. R. Fuller and F. J. Fahy, "Characteristics of wave propagation and energy distributions in cylindrical elastic shells filled with fluid," J. Sound Vib. 81, 501-518 (1982).

${ }^{24}$ B. E. Abulnaga, Slurry Systems Handbook (McGraw-Hill, New York, 2002).

${ }^{25}$ S. R. Addison, "Study of sound attenuation in sediments," Ph.D. dissertation, University of Mississippi, 1984.

${ }^{26}$ A. D. Pierce, Acoustics: An Introduction to its Physical Principles and Applications (American Institute of Physics, Woodbury, NY, 1994). 